\title{
Extending international access to doubly-labelled water data in older adults
}

\author{
J. Porter ${ }^{1,2}$, K. Nguo ${ }^{1,3}$, S. Gibson ${ }^{1}$, C.E. Huggins ${ }^{1}$, J. Collins ${ }^{1,4}$, N.J. Kellow ${ }^{1}$ and H. Truby ${ }^{1}$ \\ ${ }^{1}$ Department of Nutrition, Dietetics \& Food, Monash University, ${ }^{2}$ Allied Health Clinical Research Office, Eastern \\ Health, ${ }^{3}$ Department of Nutrition \& Food Services, Royal Children's Hospital and ${ }^{4}$ Dietetics Department, \\ Eastern Health, Australia.
}

Impacts on social, economic and health systems that result from global ageing have been well documented ${ }^{(1,2)}$. It is important that evidence for total energy expenditure (TEE) in this older population is synthesised, interpreted, and applied to improve nutrition and health outcomes at a population, community and individual level. Doubly-labelled water (DLW) is recognised as the gold standard method for measuring TEE. In the first stage of a multi-phase study, we aimed to establish the extent of TEE data measured by DLW in the population of older adults aged $\geqslant 65$ years, and to make recommendations for data sharing ${ }^{(3)}$.

Participant level TEE data measured by DLW were identified using systematic review principles; the protocol was registered (PROSPERO registration CRD42016047549). Studies were eligible where all participants were aged $\geqslant 65$ years, or where participantlevel data could be obtained for those $\geqslant 65$ years. No restrictions were placed on date, language or study designs of original research; review and conference abstracts were ineligible. The search strategy was devised in consultation with a medical librarian. Four databases (MEDLINE complete, EMBASE, CINAHL Plus, and Cochrane Central) were searched from inception to July 2016, complemented by hand searching. Title and abstract screening then full text review was undertaken independently and in duplicate. Where participant level data were not publicly accessible, attempts were made to contact the corresponding author by email to request access to data.

The database search yielded 1491 records with another 5 identified through other sources. Following removal of duplicates and the title/abstract screen, 317 full text papers were reviewed. The final library included 45 publications of 28 studies; many were ineligible due to the wrong population (e.g. age <65years) and the wrong outcome (e.g. DLW not used to measure energy expenditure). Data were reported in fifteen publications and data were contributed directly from authors of 30 publications with the final library including 45 publications of 28 studies. In total, participant level data were accessible for 890 participants aged $\geqslant 65$ years. Approximately $67 \%$ of TEE data measured by DLW for the population of interest were unable to be obtained. Figure 1 describes several approaches that should be considered to increase DLW data access.

1. Depositing all data as part of the published article, either within the paper or as a supplemental file

2. Development of a nutrition specific data repository

3. Utilising pre-existing data repositories, including clinical research or university data repositories

4. A minimum set of parameters should be developed to enable consistency in the deposited data.

Fig. 1. Opportunities for international sharing of doubly-labelled water data.

In conclusion, the systematic approach used to establish the extent of TEE data has identified the need for international data repositories that aim to minimise the loss of access to data over time. Greater transparency in reporting primary data would support the international research effort.

1. Tinker A. (2002). Mech Ageing Dev 123, 729-735.

2. Andrews GR. (2001) Br J Nutr 322, 728-729.

3. Porter J, Nguo K, Gibson S et al. (2018) Nutrition Journal, in press. 